\title{
CONSTRUCTIBILITY OF THE SET OF POINTS WHERE A COMPLEX ANALYTIC MORPHISM IS OPEN
}

\author{
ADAM PARUSIŃSKI
}

(Communicated by Clifford J. Earle)

\begin{abstract}
Using the local flattening theorem we prove that the set of points where a complex analytic morphism is open (or universally open) is constructible. A similar result is obtained for a morphism of complex algebraic varieties.
\end{abstract}

Let $f: X \rightarrow Y$ be a morphism of schemes and let $L(f) \subseteq X$ be the set of points where $f$ is universally open (see Definition 1.2). In [EGA, (14.3.9)] Grothendieck asked whether $L(f)$ is always a constructible subset of $X$. If $X$ and $Y$ are of finite type over a field $k$ and $f$ is a morphism over $k$, then the answer is positive and was given by Roland Huber [Hu], who also posed the question whether the same is true in the complex analytic category. We shall show in $\S 1$ of this paper that this is the case (Theorem 1.4). The idea of our proof is different from that of Huber. His proof is based on the properties of real closure of a field and real spectrum (or valuation spectrum in the case of a field of positive characteristic) and ours uses flatness, namely, the local flattening theorem [HLT, Théorème 4; $\mathrm{H} 1$, Theorem 4.4].

In $\S 2$ we show how to extend our proof to the category of complex algebraic varieties. At the end we give two interesting examples of $L(f)$.

In view of Hubert's proof it is interesting to ask a similar question in the real analytic category.

Question. Let $f: X \rightarrow Y$ be a morphism of real analytic spaces. Is $L(f)$ always a semi-analytic subset of $X$ ?

As Huber pointed out to me, it is easy to see that in this case $L(f)$ is a subanalytic subset of $X$.

\section{Complex ANAlytic Case}

Definition 1.1. Let $f: V \rightarrow W$ be a continuous map of topological spaces. We say that $f$ is open at $x \in V$ if the image of each neighbourhood of $x$ is a neighbourhood of $f(x)$.

Note that the above condition does not mean that the image of any open neighbourhood of $x$ is an open (!) neighbourhood of $f(x)$. If $f$ is open at

Received by the editors May 28, 1991.

1991 Mathematics Subject Classification. Primary 32C15, 14E40.

Key words and phrases. Constructible sets, flattening theorem, universally open. 
every point of $X$, then $f$ is open (i.e., the image of an open set is open).

Definition 1.2. We say that a morphism (in some category) $f: X \rightarrow Y$ is universally open at $x \in X$ if for each morphism $\pi: \widetilde{Y} \rightarrow Y$ (in the same category) the induced morphism $\tilde{f}: X \times_{Y} \tilde{Y} \rightarrow \tilde{Y}$ is open at every point corresponding to $x$.

The following observation is basically due to Huber [Hu].

Lemma 1.3. In the category of complex or real analytic spaces and analytic morphisms the following conditions are equivalent:

(1) $f: X \rightarrow Y$ is open at $x \in X$;

(2) $f: X \rightarrow Y$ is universally open at $x \in X$;

(3) Every germ of real analytic curve $\gamma(t):([0, \varepsilon), 0) \rightarrow(Y, f(x))$ can be lifted (set theoretically) to a germ of real analytic curve $\gamma^{\prime}(t):([0, \varepsilon), 0) \rightarrow$ $(X, x)$.

Proof. (1) $\Leftrightarrow(3)$ follows from the curve selection lemma for subanalytic sets $([\mathrm{H} 1,(8.4)]$ or $[\mathrm{DLS}])$. Of course $(2) \Rightarrow(1)$.

We shall show $(1) \Rightarrow(2)$ and let $\pi: \widetilde{Y} \rightarrow Y$ be any morphism and consider the diagram

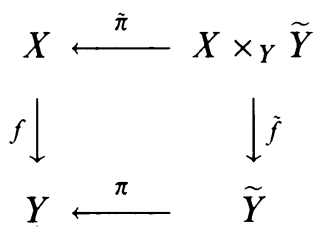

Let $\tilde{x} \in X \times_{Y} \tilde{Y}$ be such that $\tilde{\pi}(\tilde{x})=x$. Let $\tilde{\gamma}:([0, \varepsilon), 0) \rightarrow(\tilde{Y}, \tilde{f}(\tilde{x}))$ be a germ of a real analytic curve. Then, thanks to $(1) \Leftrightarrow(3)$, we can lift $\gamma=\pi(\tilde{\gamma})$ to $\gamma^{\prime}:([0, \varepsilon), 0) \rightarrow(X, x)$ (if $\gamma$ is constant equal to $f(x)$ we choose $\gamma^{\prime}$ constant

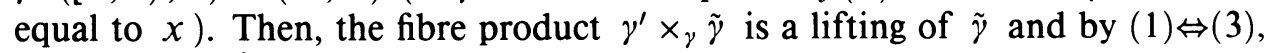
we conclude $\tilde{f}$ is open at $\tilde{x}$. This ends the proof.

For a morphism $f: X \rightarrow Y$ we denote by $L(f)$ the set of points of $X$ at which $f$ is open and by $T(f)$ the complement of $L(f)$. For an arbitrary morphism of complex analytic spaces $L(f)$ could be neither open nor closed (see Examples 1 and 2 at the end of this paper and [EGA, (14.1.3)]) but we shall show that it has to be constructible.

Theorem 1.4. Let $f: X \rightarrow Y$ be a morphism of complex analytic spaces. Then the set $L(f)$ of those points of $X$ where $f$ is universally open (= open, by Lemma 1.3) is constructible.

The proof is based on the following idea. First we change the base of $f$ by a proper surjective morphism $\pi: \widetilde{Y} \rightarrow Y$ such that the theorem holds for the induced morphism $\tilde{f}: X \times_{Y} \tilde{Y} \rightarrow \tilde{Y}$. Then we apply

Lemma 1.5 (compare [EGA, (14.4.8.1)]). Let $f: X \rightarrow Y$ be as above and let $\pi: \widetilde{Y} \rightarrow Y$ be a proper surjective morphism. Let $\tilde{f}: X \times_{Y} \widetilde{Y} \rightarrow \widetilde{Y}, \tilde{\pi}: X \times_{Y} \widetilde{Y} \rightarrow X$ denote the standard projections. Then $T(f)=\tilde{\pi}(T(\tilde{f}))$.

In particular, if $T(\tilde{f})$ is constructible so is $T(f)$.

Proof. Since $f$ is universally open outside $T(f)$ (Lemma 1.3) we have $T(f) \supseteq$ $\tilde{\pi}(T(\tilde{f}))$. Next we show the converse. 
Since $\pi$ is proper so is $\tilde{\pi}$. Therefore the inverse images of a fundamental system of neighbourhoods of $x \in X$ form a fundamental system of neighbourhoods of $\tilde{\pi}^{-1}(x)$.

Let $x \in X \backslash \tilde{\pi}(T(\tilde{f}))$ and let $U_{x}$ be a neighbourhood of $x$ in $X$. We shall show that $f\left(U_{x}\right)$ is a neighbourhood of $y=f(x)$. Since $\tilde{f}$ is open at every point of $\tilde{\pi}^{-1}(x), \tilde{f}\left(\tilde{\pi}^{-1}\left(U_{x}\right)\right)$ is a neighbourhood of $\tilde{f}\left(\tilde{\pi}^{-1}(x)\right)=\pi^{-1}(y)$. Again, since $\pi$ is proper, the image by $\pi$ of any neighbourhood of $\pi^{-1}(y)$ is a neighbourhood of $y$. Therefore $\pi\left(\tilde{f}\left(\tilde{\pi}^{-1}\left(U_{x}\right)\right)\right)=f\left(U_{x}\right)$ is a neighbourhood of $y$. Thus $T(f) \subseteq \tilde{\pi}(T(\tilde{f}))$. This ends the proof of the lemma.

Proof of Theorem 1.4 for proper morphisms. If $f: X \rightarrow Y$ is proper and $Y$ is reduced then we can flatten it using the global flattening theorem [H3, Theorem 4.4]. Namely, there exists a proper morphism $\pi: Y^{\prime} \rightarrow Y$, which is the composition of a locally finite family of blowings-up with nowhere dense centres, such that the strict transform $f^{\prime}: X^{\prime} \rightarrow X$ of $f$ by $\pi$ is flat. By definition of strict transform, $X^{\prime}$ is the smallest analytic subspace of $X \times_{Y} Y^{\prime}$ that contains $X \times_{Y}\left(Y^{\prime}-E\right)$, where $E$ denotes the union of corresponding exceptional divisors of $\pi$, and $f^{\prime}$ is induced by the projection on the first factor. Therefore, set theoretically $X \times_{Y} Y^{\prime}=X^{\prime} \cup X^{\prime \prime}$, where $\tilde{f}\left(X^{\prime \prime}\right)$ is contained in $E$ and

$$
T(\tilde{f})=T\left(f^{\prime}\right) \cup\left(X^{\prime \prime} \backslash X^{\prime}\right),
$$

where again $\tilde{f}: X \times_{Y} Y^{\prime} \rightarrow Y^{\prime}$ denotes the projection. Since the flat maps are open (see, e.g., [F, 3.19]), $T\left(f^{\prime}\right)=\varnothing$ and consequently $T(\tilde{f})$ is constructible. Hence, by Lemma 1.5, $T(f)$ is constructible as desired.

We remark that in the proof the flatness could be replaced by a weaker property such as, for instance, equidimensionality. We leave the proof of the following fact to the reader.

Remark 1.6 (Chevalley's criterion for openness [EGA, (14.4.4)]). Let $f: X \rightarrow Y$ be as above. Then $f$ is open at $x$ if the following property, called equidimensionality at $x$ holds: for each irreducible component $Y^{\prime}$ of $(Y, y)$, where $y=f(x)$,

$$
\operatorname{dim}\left(f^{-1}(y), x\right)=\operatorname{dim}\left(f^{-1}\left(Y^{\prime}\right), x\right)-\operatorname{dim}\left(Y^{\prime}, y\right) .
$$

To flatten a nonproper morphism in place of blowings-up we shall use compositions of local blowings-up. We recall that a local blowing-up $\widetilde{U} \rightarrow U \hookrightarrow Y$ is the composition of an open embedding $U \hookrightarrow Y$ and a blowing-up $\widetilde{U} \rightarrow U$.

Theorem 1.7 (Local flattening theorem [HLT, Théorème 4; H1, Theorem 4.4]). Let $f: X \rightarrow Y$ be a morphism of complex analytic spaces and assume that $Y$ is reduced. Let $y \in Y$ and let $L$ be a compact subset of $f^{-1}(y)$. Then there exists a finite number of morphisms $\pi_{\alpha}: Y_{\alpha} \rightarrow Y$ such that each of them is the composition of (finitely many) local blowings-up with nowhere dense centres and

(i) for every $\alpha$, there is a compact subset $K_{\alpha}$ of $Y_{\alpha}$ such that $\bigcup_{\alpha} \pi_{\alpha}\left(K_{\alpha}\right)$ is a (compact) neighbourhood of $y$ in $Y$.

(ii) for every $\alpha$, the strict transform $f_{\alpha}: X_{\alpha} \rightarrow Y_{\alpha}$ of $f$ by $\pi_{\alpha}$ is flat at every point corresponding to $L$. 
Remark 1.8. For our purpose we can replace (ii) by a weaker condition:

(ii') for every $\alpha$, the strict transform $f_{\alpha}: X_{\alpha} \rightarrow Y_{\alpha}$ of $f$ by $\pi_{\alpha}$ is equidimensional in a neighbourhood of the set of points corresponding to $L$.

See [P, Corollary 1.2] for the proof of such a weaker version of Theorem 1.7 (where it is proven under an additional assumption that $Y$ is nonsingular but the proof there can be easily adapted to arbitrary $Y$ or we can resolve first the singularities of $Y$ using [H2] or [BM]).

Proof of Theorem 1.4 (general case). Fix $x \in X$. We shall show that the germ $(T(f), x)$ is constructible.

By Theorem 1.7 there is a family $\left\{\pi_{\alpha}: Y_{\alpha} \rightarrow Y\right\}$ of compositions of local blowings-up with nowhere dense centres satisfying (i) and (ii) for $L=\{x\}$. Since local blowings-up are not proper in general we cannot use Lemma 1.5 directly. Instead, we proceed by induction on the total number $n(x)$ of local blowings-up in $\left\{\pi_{\alpha}\right\}$. If $n(x)=0$ then $f$ is flat at $x$ and $(T(f), x)$ is empty.

Note that we can pull back the family $\left\{\pi_{\alpha}\right\}$ by a local blowing-up without increasing the total number of blowings-up. Indeed

(a) Let $U$ be a neighbourhood of $y=f(x)$ and let $K$ be a compact subneighbourhood of $y$ in $U$. Then $\left\{\left.\pi_{\alpha}\right|_{\pi_{\alpha}^{-1}(U)}\right\}$ and $\left.\left\{K_{\alpha} \cap \pi_{\alpha}^{-1}(K)\right)\right\}$ satisfies the statement of Theorem 1.7 for $\left.f\right|_{f^{-1}(U)}$. The centres of local blowings-up in $\left.\pi_{\alpha}\right|_{\pi_{\alpha}^{-1}(U)}$ are open subsets of those of $\pi_{\alpha}$.

(b) Let $\sigma: Y^{\prime} \rightarrow Y$ be a blowing-up with nowhere dense centre. Then, by $[\mathrm{H} 1,(2.16)]$ the strict transforms $\pi_{\alpha}^{\prime}: Y_{\alpha}^{\prime} \rightarrow Y^{\prime}$ of $\pi_{\alpha}$ by $\sigma$ are compositions of local blowings-up with nowhere dense centres (and these centres correspond to the ones of $\pi_{\alpha}$ ). Let $f_{\alpha}^{\prime}: X_{\alpha}^{\prime} \rightarrow Y_{\alpha}^{\prime}$ be the strict transform of $f^{\prime}$ by $\pi_{\alpha}^{\prime}$ and let $\sigma_{\alpha}: Y_{\alpha}^{\prime} \rightarrow Y_{\alpha}, \sigma^{\prime}: X^{\prime} \rightarrow X$ be the induced proper maps. Then, $\left\{\pi_{\alpha}^{\prime}\right\}$ and $\left\{K_{\alpha}^{\prime}=\sigma_{\alpha}^{-1}\left(K_{\alpha}\right)\right\}$ satisfy the statement of Theorem 1.7 for the strict transform $f^{\prime}$ of $f$ by $\sigma$ and $L^{\prime}=\sigma^{-1}(L)$.

(c) By (a), the point (b) holds also if $\sigma$ is a local blowing-up with nowhere dense centre.

Inductive step. Let $\sigma: Y^{\prime} \rightarrow Y$ be the first blowing-up in one of $\pi_{\alpha}$, say $\pi_{\alpha_{0}}$. By (a), localizing around $y$, we can assume that $\sigma$ is a blowing-up. Let us now follow the notation of (b) above. We claim that the total number of local blowings-up in the family $\left\{\pi_{\alpha}^{\prime}\right\}$ is smaller than $n(x)$. In fact, the first blowing-up in $\pi_{\alpha_{0}}^{\prime}$ is the strict transform of $\sigma$ by $\sigma$ so it is the identity. Therefore, by the inductive assumption, $\left(T\left(f^{\prime}\right), x^{\prime}\right)$ is constructible for any $x^{\prime} \in L^{\prime}$. Hence the germ $\left(T\left(f^{\prime}\right), L^{\prime}\right)$ is constructible and, since $\sigma^{\prime}$ is proper and by Lemma 1.5, we conclude that $(T(f), x)$ is constructible. This ends the proof of Theorem 1.4.

In general the converse of Chevalley's criterion for openness is not true (Examples 1 and 2). Nevertheless, we show below that it holds for a generic point of the fibre.

Proposition 1.9. Let $f: X \rightarrow Y$ be as above and assume for simplicity that $X$ 
nontrivial morphism $\tau: S \rightarrow Y$, where $S$ is a curve and $y \in \operatorname{Im}(\tau)$. Then, the set of points of $f^{-1}(y)$, where the induced morphism $X \times_{Y} S \rightarrow S$ is open, is of dimension $\operatorname{dim} X-\operatorname{dim} Y$. By Lemma 1.3, the set of points of $f^{-1}(y)$, where $f$ is open cannot be bigger, and thus we get the conclusion.

\section{REMARKS ON THE COMPLEX ALGEBRAIC CASE}

We show that Theorem 1.4 holds in the category of complex algebraic varieties. Thanks to the following observation $[\mathrm{Hu}]$, it is the same to consider Zariski or strong topology.

Lemma 2.1. Let $f: X \rightarrow Y$ be a regular morphism of complex algebraic varieties. Then the following conditions are equivalent:

(1) $f: X \rightarrow Y$ is (Zariski) open at $x \in X$;

(2) $f: X \rightarrow Y$ is (Zariski) universally open at $x \in X$;

(3) $f: X \rightarrow Y$ is open (in the strong topology) at $x \in X$;

(4) $f: X \rightarrow Y$ is universally open (in the strong topology) at $x \in X$.

Proof. (3) $\Leftrightarrow(4)$ follows from Lemma 1.3. (1) $\Leftrightarrow(3)$ will follow from the proof of Theorem 2.2. $(2) \Rightarrow(1)$ is immediate.

We sketch the proof of $(1) \Rightarrow(2)$. First note that it suffices to check the universal openness only for the change of base $\pi:\left(\tilde{Y}, \tilde{y}_{0}\right) \rightarrow(Y, f(x))$, where $\widetilde{Y}$ is an irreducible curve. Then, if $\pi$ is not constant, it has finite fibres and so has $\tilde{\pi}: X \times_{Y} \tilde{Y} \rightarrow X$. Let $\tilde{x}=\left(x, \tilde{y}_{0}\right)$. Then, the inverse image of a base of neighbourhoods of $x$ is a base of neighbourhoods of $\tilde{x}$. Therefore, if $f$ is open at $x$, then $\tilde{f}: X \times_{Y} \widetilde{Y} \rightarrow \tilde{Y}$ is open at $\tilde{x}$.

Theorem 2.2. Let $f: X \rightarrow Y$ be a regular morphism of complex algebraic varieties. Then the set $L(f)$ of those points of $X$ where $f$ is universally open (in strong or Zariski topology) is constructible.

Proof of Theorem 2.2. The proof will be for both topologies simultaneously.

We proceed exactly in the same way as in the proof of Theorem 1.4 for proper morphisms. Instead of the global flattening theorem we use Theorem 1 of [R, Chapter 4] that says we can flatten $f$ by blowing up an algebraic nowhere dense centre (assuming $Y$ is reduced). Let $\pi: Y^{\prime} \rightarrow Y$ be such blowing-up and let $\tilde{f}: X \times_{Y} Y^{\prime} \rightarrow X$ be the induced morphism. Then $T(\tilde{f})$ is constructible: in the strong topology by Lemma 1.5 and by its algebraic version [EGA, (14.4.8.1)] in the Zariski topology, so is $T(f)=\tilde{\pi}(T(\tilde{f})$. This ends the proof.

In the proof above, the set of points $T(\tilde{f})$ where $\tilde{f}$ is not open is the same for both Zariski and strong topology. Therefore the same holds for $T(f)=$ $\tilde{\pi}(T(\tilde{f}))$. This shows the equivalence $(1) \Leftrightarrow(2)$ in Lemma 2.1 .

Example 1. Let $f: \mathbf{C}^{2} \rightarrow \mathbf{C}^{2}$ be given by $f(x, y)=\left((x+y) x^{2},(x+y) y^{2}\right)$. Then $f$ is open at the origin although $\operatorname{dim}\left(f^{-1}(0), 0\right)>0$.

Indeed, if $(p, q) \neq 0$, then

$$
f^{-1}(p, q)=\left( \pm p^{1 / 2}+ \pm q^{1 / 2}\right)^{-1 / 3}\left( \pm p^{1 / 2}, \pm q^{1 / 2}\right)
$$


and we can always choose the signs in such a way that $\left|\left( \pm p^{1 / 2}+ \pm q^{1 / 2}\right)\right| \geq$ $\left|\left( \pm p^{1 / 2}, \pm q^{1 / 2}\right)\right|$ and therefore we can always find $(x, y) \in f^{-1}(p, q)$ such that $|(x, y)| \leq\left|\left( \pm p^{1 / 2}, \pm q^{1 / 2}\right)\right|^{2 / 3}$. It is easy to see that $L(f)=\left(\mathbf{C}^{2} \backslash f^{-1}(0)\right) \cup\{0\}$.

Example 2. Let $X \subseteq \mathbf{C}^{4}$ be the union of two coordinate planes

$$
\begin{aligned}
& X_{1}=\left\{(w, x, y, z) \in \mathbf{C}^{4} ; w=x=0\right\} \\
& X_{2}=\left\{(w, x, y, z) \in \mathbf{C}^{4} ; y=z=0\right\}
\end{aligned}
$$

and let $f: X \rightarrow \mathbf{C}^{2}$ be given by

$$
\begin{gathered}
f_{\mid X_{1}}(y, z)=((y+z) y,(y+z) z), \\
f_{\mid X_{2}}(w, x)=((w-x) w,(w-x) x) .
\end{gathered}
$$

Then neither $f_{\mid X_{1}}$ nor $f_{\mid X_{2}}$ is open at the origin but $f$ is. Indeed,

$$
\begin{aligned}
& \left(f_{\mid X_{1}}\right)^{-1}(p, q)=\left( \pm(p+q)^{-1 / 2}(p, q),\right. \\
& \left(f_{\mid X_{2}}\right)^{-1}(p, q)=\left( \pm(p-q)^{-1 / 2}(p, q)\right.
\end{aligned}
$$

and therefore we can always find $(x, y) \in f^{-1}(p, q)$ such that $|(x, y)| \leq$ $|(p, q)|^{1 / 2}$. It is easy to see that $L(f)=\left(X \backslash f^{-1}(0)\right) \cup\{0\}$.

\section{ACKNOWLEDGMENTS}

I would like to thank Roland Huber for many valuable suggestions and remarks concerning this paper. The first version of this paper was written during my stay at the Max-Planck-Institut für Mathematik as a fellow of the Alexander von Humboldt Foundation. I would like to express my gratitude to the institute for the warm hospitality and to the foundation for its financial support.

\section{REFERENCES}

[BM] E. Bierstone and P. D. Milman, Uniformization of analytic spaces, J. Amer. Math. Soc. 24 (1989), 801-836.

[DLS] Z. Denkowska, S. Lojasiewicz, and J. Stasica, Certaines propriétés élémentaires des ensembles sous-analytiques, Bull. Acad. Polon. Sci. Sér. Sci. Math. Astronom. Phys. 27 (1979), 529-535.

[EGA] A. Grothendieck and J. Dieudonne, Éléments de géométrie algébrique. IV, Inst. Hautes Études Sci. Publ. Math. 32 (1967).

[F] G. Fisher, Complex analytic geometry, Lecture Notes in Math., vol. 538, Springer-Verlag, Berlin and Heidelberg, 1976.

[H1] H. Hironaka, Introduction to real analytic sets and real-analytic maps, Instituto “L.Tonelli", Pisa, 1973.

[H2] Resolution of singularities of an algebraic variety over a field of characteristic zero. I, II, Ann. of Math. (2) 79 (1964), 109-326.

[H3] _ Flattening theorem in complex analytic geometry, Amer. J. Math. 97 (1975), 199-265.

[H-L-T] H. Hironaka, M. Lejeune-Jalabert, and B. Teissier, Platificateur local en géométrie analytique et aplatissement local, Astérisque 7-8 (1973), 441-446 . 
[Hu] R. Huber, Two questions about open mappings, talk at conference "Reelle algebraische Geometrie" Oberwolfach, June 10-16, 1990.

[P] A. Parusiński, Subanalytic functions, preprint MPI/90-58; Trans. Amer. Math. Soc. (to appear).

[R] M. Raynaud, Flat modules in algebraic geometry, Compositio Math. 24 (1972), 11-31.

Institute of Mathematics, Gdańsk University, ul.Wita Stwosza 57, PL-80-952 Gdańsk, Poland

Current address: School of Mathematics and Statistics, The University of Sydney, Sydney, New South Wales 2006, Australia

E-mail address: parusinski_a@ math.su.oz.au 